(C) 2018. This manuscript version is made available under the CC-BY-NC-ND 4.0 license http://creativecommons.org/licenses/by-nc-nd/4.0/

\title{
Sensitivity enhancement experimental demonstration using a low cutoff wavelength SMS modified structure coated with a pH sensitive film.
}

Authors: W. E. Rodríguez-Rodríguez ${ }^{1 *}$, I. Del Villar-Fernandez ${ }^{2}$, C. Ruiz-Zamarreño, I. R. Matías-Maestro' ${ }^{2}$, F. J. Arregui-San Martin ${ }^{2}$, A. J. Rodríguez- Rodríguez ${ }^{3}$, R. F. DomínguezCruz $^{1}$.

\section{Affiliations:}

1 Unidad Académica Multidisciplinaria Reynosa-Rodhe. Universidad Autónoma de Tamaulipas. Reynosa-San Fernando Highway, 88779. Reynosa, México.

2 Electrical and Electronic Engineering Department, Universidad Pública de Navarra, Edif. Los Tejos, Campus Arrosadia, 31006 Pamplona, Spain.

*Corresponding author:

Wenceslao Eduardo Rodríguez: wrodriguez@uat.edu.mx

Abstract: The multimode interference (MMI) effect in a single mode-multimode-single mode (SMS) can be used for development of wavelength shift detection based sensors. In this work, the focus is centered on obtaining wavelength shifts with low cutoff single mode fibers, which allows exploring the wavelength range from 600 to $1000 \mathrm{~nm}$, where optical sources and detectors are less expensive than at longer wavelengths. In addition, the application of a reduction in the fiber diameter of the SMS structure by means of HF etching, combined with the deposition of a thin-film, enables to enhance the sensitivity of the devices at the same time the objective mentioned before is achieved. In this sense, the effect of the deposition of a pH sensitive thin-film on SMS structures with different diameters allowed attaining a maximum sensitivity of $15 \mathrm{~nm}$ per $\mathrm{pH}$ unit in the range from $\mathrm{pH} 4$ to $\mathrm{pH} 6$, which improves by a factor of 3 the sensitivity of SMS sensors without etching operating at longer wavelengths.

Keywords: single mode-multi mode-single mode (SMS), etching, optical fiber sensors, pH sensor. 


\section{INTRODUCTION}

During the last 30 years numerous optical fiber optic sensors have been developed to monitor different physical variables such as temperature, strain and refractive index [1]. Optical fiber presents interesting properties: electromagnetic immunity, low attenuation and compact dimensions. In addition, multiple configurations have been explored, such as those based on photonic crystal fibers, optical fiber long period gratings (LPGs), optical fiber Bragg gratings (FBGs) or optical fiber interferometers based on Fabry-Pérot, Mach-Zender and Michelson configurations [2, 3]. Fiber optic sensors are used in different fields, such as civil engineering in structural health monitoring, or the gas, oil and renewable energy industries to control and reduce the pollution emissions to the environment [4].

Optical fibers sensors can be divided into two different categories: extrinsic and intrinsic. In the first one, the light beam is modulated outside the fiber for the measurement using: mirrors, gas, liquid or other mechanisms. In the intrinsic method, the light signal is modulated within the fiber [1].

A low-cost fabrication alternative technique based on the intrinsic method consists of the multi modal interference (MMI) configuration. MMI configuration has been widely explored with the utilization of single mode-multimode-single mode (SMS) structures for temperature, strain, displacement and refractive index sensing applications $[5,6]$. This phenomenon can be implemented using a simple SMS structure, which is fabricated by means of splicing two segments of single mode fiber (SMF) at the ends of a multi-mode fiber (MMF) segment [7-9]. With this structure, different maxima and minima are created in the optical spectrum. Most of the research in this kind of optical sensors has been focused on wavelengths longer than $1000 \mathrm{~nm}$. This requires the utilization of complex and expensive light sources and detectors, which reduces the utilization of these devices in many applications. Therefore, the implementation of a SMS device that operates at shorter wavelengths in the range of $600-1000 \mathrm{~nm}$, where optical sources and detectors are less expensive, could help to widen the fields of application of these devices. A main drawback of the application of these devices at shorter wavelengths is that the sensitivity is reduced 
[10]. Consequently, it is necessary to improve the sensitivity of these devices in the visible and near infrared range to be considered as useful tools for sensing applications.

In the next sections, we will describe the implementation of a low-cost optical fiber sensing alternative using SMS structures with enhanced sensitivity in the visible and near infrared region. As an example of application of the proposed device, a pH sensor will be developed and tested for different diameters. Other optical fiber $\mathrm{pH}$ sensors have been developed [11, $12,13]$; but these devices operate in the infrared region and they need an expensive experimental setup. In this paper, we present a less expensive $\mathrm{pH}$ sensor with higher sensitivity by unit compared to the previously cited researches. Particularly, the optical fiber $\mathrm{pH}$ sensors can be applied in: biochemistry [14, 15], structural monitoring [16] and energy $[17,18,19]$.

\section{SMS STRUCTURE: MODES OF OPERATION.}

The SMS structure is developed by fusion splicing a section of no-core multimode fiber (MMF) between two SMF pigtails. The incident light is transmitted through the core of the SMF and coupled into different propagation modes in a segment of MMF [9]. At the end of the MMF segment the light is recoupled into the core mode of another SMF.

Fig. 1. Single mode-multi mode-single mode structure.

The self-imaging effect it is an interesting phenomenon which allows to understand the performance of an SMS structure $[7,20]$. When an optical signal is launched into an SMS structure, it excites the supported modes of the MMF. As light propagates through the MMF, images of the input fields are created at specific lengths. Due to the MMI effect, both transmission and attenuation bands are obtained in the optical spectrum. At certain lengths, where the phase difference is multiple of $2 \pi$, self-images are obtained, exact replicas of the input field $[20,21]$. The transmission bands obtained by the self-imaging effect can be controlled by the dimension of the MMF segment. The length of the MMF section for positioning the band at a specific wavelength can be obtained with this expression [22]: 


$$
Z_{i}=\frac{p D^{2} n_{\text {core }}}{\lambda}
$$

where $D$ is the diameter of the structure, $n_{\text {core }}$ is the refractive index of MMF, $\lambda$ is the operational wavelength and $p$ is the self-image order.

As an example, Fig. 2(a) shows the transmission spectrum of an SMS structure with diameter $125 \mu \mathrm{m}$ and MMF length $60 \mathrm{~mm}$. Coreless MMF segments from POFC Inc. (Taiwan) and standard SMF pigtails from Telnet Redes Inteligentes Inc. (Zaragoza, Spain) were used for manufacturing this structure. Since standard SMF fiber is designed for operation at wavelengths above $1100 \mathrm{~nm}$, the wavelength range analyzed was 1150-1700 nm. In Fig. 2(a) the self-image band can be observed at $1500 \mathrm{~nm}$. However, it has been proved more recently that for small diameters it is possible to obtain a sinusoidal spectrum (see Fig. 2(b) for an SMS structure with diameter $30 \mu \mathrm{m}$ and MMF length $60 \mathrm{~mm}$ ), where the sensitivity to the surrounding refractive index is improved thanks to the diameter reduction $[11,23]$. Indeed, the sensitivity does not depend on the monitorization of a self-image band, but rather on the diameter of the device. This sensitivity increased is explained by the fact that the position of the transmission bands is dependent on the effective indices of modes in the MMF section [24], and it is well known that as the diameter of an optical fiber is reduced, the effective index of the modes becomes more sensitive to the surrounding medium refractive index [25].

Fig. 2. Transmission spectrum for: a) SMS structure with diameter $125 \mu \mathrm{m}$ and MMF length $60 \mathrm{~mm}$; b) SMS structure with diameter $25 \mu \mathrm{m}$ and MMF length $60 \mathrm{~mm}$.

\section{CHARACTERIZATION OF LOW CUTOFF WAVELENGTH ETCHED SMS STRUCTURES}

\section{A. Experimental setup}

To operate at shorter wavelengths than those explored in Fig. 2, the SMS structure under analysis consisted of a $20 \mathrm{~mm}$ coreless fiber segment, spliced on each end to an SMF S630 Thorlabs (core diameter $3.5 \mu \mathrm{m}$ and cladding diameter $125 \mu \mathrm{m}, \mathrm{NA}=0.12$ and operating wavelength $630-860 \mathrm{~nm})$. The SMS structure was kept straight during both the construction 
and the characterization processes to avoid any bending artifacts. To this purpose, the Uholder depicted in Fig. 3 was used to support the SMS structure during the etching process. Since the operating wavelength range is located below $1100 \mathrm{~nm}$, an ASBN-W tungstenhalogen broadband source from Spectral Products Inc. and an HR4000 spectrometer from Ocean Optics were used (see Fig. 3). In Fig. 4 the SMS structure after the $40 \%$ HF etching is depicted.

Fig. 3. Experimental setup for monitoring the optical spectrum of the SMS structure during the etching process.

\section{B. Diameter reduction}

Before explaining the diameter reduction process, it is important to identify the built Etched-SMS structures: E-SMS1 $=65 \mu \mathrm{m}, \mathrm{E}-\mathrm{SMS} 2=45 \mu \mathrm{m}$ and E-SMS3 $=25 \mu \mathrm{m}$; depicted in Fig.4. Each E-SMS structure was deposited in 40\% HF during: E-SMS1 = 30 minutes, E-SMS2 $=40$ minutes and $\mathrm{E}-\mathrm{SMS} 3=50$ minutes. After that, it is necessary to remove the corresponding E-SMS structure from the HF solution and to clean with water afterwards. Finally, the structures are left in air for at least 6 hours.

Fig. 4. SMS structure after the $40 \% \mathrm{HF}$ etching with diameters $=65,45$ and $25 \mu \mathrm{m}$.

\section{Refractive index analysis}

To verify the response of the wavelength shifts in the E-SMS structures, the E-SMS3 structure $(25 \mu \mathrm{m})$ was immersed in three different refractive indices. Its sinusoidal spectrum (see Fig. 5) allowed tracking wavelength shifts of any of the different maxima and minima. 
Fig. 5. Transmission spectra for an etched SMS structure with $25 \mu \mathrm{m}$ diameter in three different refractive index solutions.

In order to prove the hypothesis of the research, that the reduction of the diameter in the SMS structures allow to increase the sensitivity due the HF at 40\%; the E-SMS1, E-SMS2 and E-SMS3 structures were immersed in differents refractive index solutions. Fig. 6 shows the sensitivity response for the three structures. The wavelength shift in the refractive index range 1.333-1.421 for the E-SMS1 structure was $3 \mathrm{~nm}$, for the E-SMS2 structure was $4 \mathrm{~nm}$ and for the E-SMS3 strucutre was $17 \mathrm{~nm}$. The results show that the sensitivity increases for SMS structures with a smaller diameter up to $193 \mathrm{~nm}$ per refractive index unit.

Fig. 6. Wavelength shift as a function of the refractive index for three SMS sturctures with three different diameters: 125,65 and $25 \mu \mathrm{m}$.

\section{APPLICATION OF LOW CUTOFF ETCHED SMS STRUCTURES FOR PH SENSING}

A. $p H$ sensor fabrication process

Before presenting the results for a $\mathrm{pH}$ sensing application, it is important to show the diameters of the etched-SMS structures: E-SMS1 structure $=64.15 \mu \mathrm{m}, \mathrm{E}-\mathrm{SMS} 2$ structure $=$ $41.07 \mu \mathrm{m}$ and E-SMS3 structure $=23.73 \mu \mathrm{m}$. To verify the diameter obtained by etching, the diameter of the E-SMS structures was compared with the original diameter of the SMS structure (Coreless MMF $125 \mu \mathrm{m}$ ) using a digital microscope MEIJI MT87 and a computational algorithm based on triangulation of images developed in MATLAB ${ }^{\circledR}$ (see Fig. 7).

Fig.7. a) Original diameter SMS Structure $=125 \mu \mathrm{m}$. b) E-SMS1 structure diameter $=64.15$ $\mu \mathrm{m}$. c) E-SMS2 structure diameter $=41.07 \mu \mathrm{m}$. d) E-SMS3 structure diameter $=23.73 \mu \mathrm{m}$.

After the etching process, each structure was fit to a deposition robot XYZ to deposit a thinfilm with layer-by-layer (LbL) self-assembly technique [11]. To begin the process, it was necessary to immerse an E-SMS structure into $0.1 \mathrm{M} \mathrm{KOH}$ solution for 10 min to acquire 
negative charge. Then, the E-SMS was cleaned up in ultrapure water. After that, it was subjected to alternated immersions of 2 minutes in two solutions of poly (allylamine hydrochloride) (PAH) and poly (acrylic acid) (PAA) to obtain a layer pair. The $\mathrm{pH}$ of the solutions was measured using an electronic pH-meter (Crison Inc.) and was necessary to add $\mathrm{NaOH}$ and $\mathrm{HCl}$ to adjust the solutions at $\mathrm{pH}=4.5$. Both solutions were prepared using ultrapure deionized water (18.2 $\mathrm{M} \Omega)$.

The total number of layer pairs deposited for each E-SMS structure was 4 . The selection of this value was based on the mode transition, which occurs when a thin-film is deposited on an SMS structure [24]. This phenomenon allows enhancing the sensitivity of the device if an adequate thin-film thickness is selected. An increase in the coating thickness leads to a higher sensitivity. However, at the same time there is a reduction in the visibility of the transmission bands [27]. Moreover, if the coating thickness exceeds a specific value the bands are no longer visible [24]. Consequently, it is necessary to select a coating thickness that allows attaining the maximum sensitivity at the same time the bands are still visible. In the structure explored in [27] the maximum number of bilayers deposited was 18. In view that the experiments were performed at $1500-1600 \mathrm{~nm}$, and that here the central wavelength of the band to analyze is located at about $800 \mathrm{~nm}$, the coating thickness should be reduced, proportionally, to half the number of bilayers: 9. However, according to our experiments we have observed that 4 bilayers are is the limit value that enables a precise tracking of the transmission band, as it is described in Fig. 8. Otherwise the signal is too weak (see Fig. 8). In this sense, it must be considered that light is being coupled to an optical fiber with core diameter $3.5 \mu \mathrm{m}$. 


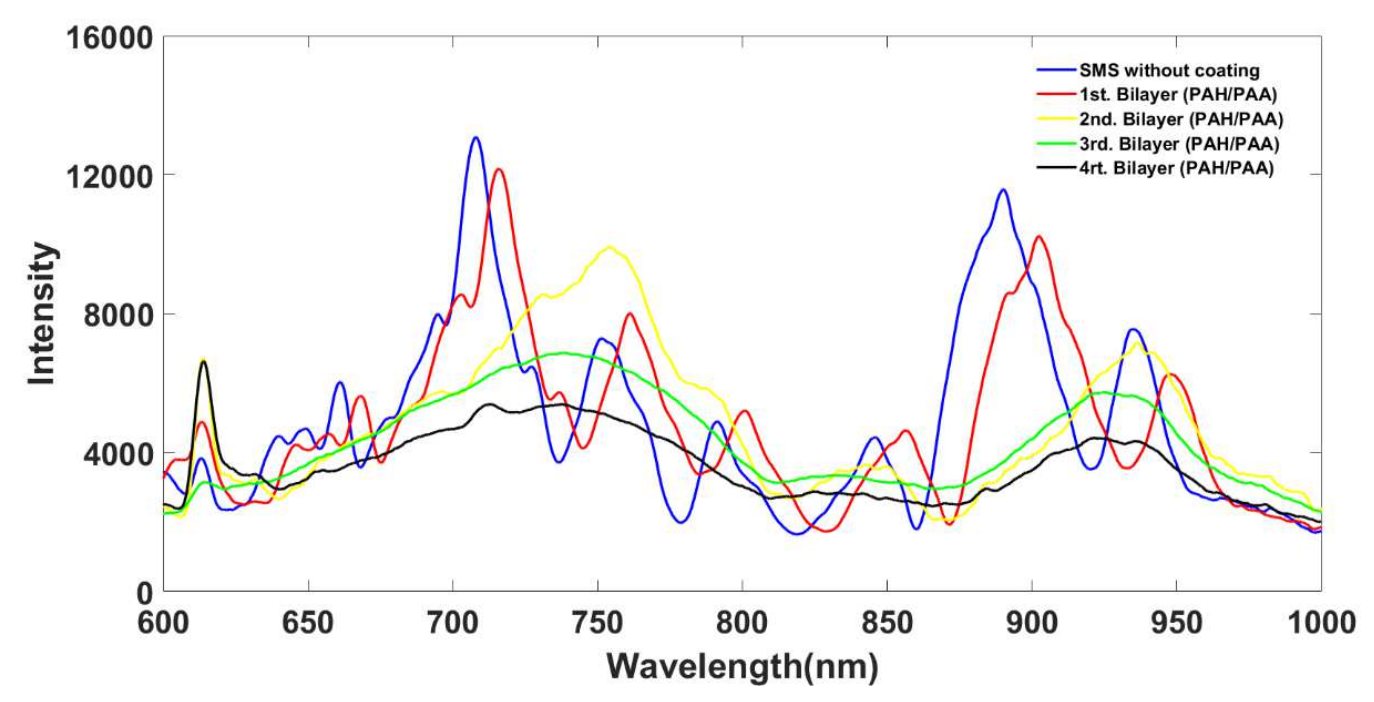

Fig. 8. Transmission spectral recorded after the deposition of each PAH/PAA bilayer.

\section{B. $p H$ characterization process}

The sensitivity response of the three E-SMS structures coated with 4 bilayers of PAH/PAA was analyzed and compared. Each E-SMS structure was immersed alternatively in pH 4 and 6 buffer solutions (60 seconds in each solution). This time allowed obtaining a stable transmission spectrum, which permitted to track correctly the central wavelength of one of the bands in the spectrum. PAH/PAA based sensors can be used for $\mathrm{pH}$ values ranging from 3 to $7[11,28]$. However, in view that the aim in this work is to see the sensitivity increase as a function of the fiber diameter, only $\mathrm{pH}$ values 4 and 6 have been explored.

Regarding the E-SMS1 structure, the central wavelength of the band monitored during the experiment experiences a wavelength shift of $4.5 \mathrm{~nm}$ (see Fig. 9a). The E-SMS2 structure presented a wavelength shift in the same $\mathrm{pH}$ solutions of $15 \mathrm{~nm}$ (see Fig. 9b). Finally, a wavelength shift of $30 \mathrm{~nm}$ was attained for E-SMS3 structure (see Fig. 9c). The unstable signal between the immersion in $\mathrm{pH} 4$ and $\mathrm{pH} 6$ obeys to the rinsing in water after the extraction of the sensor from each $\mathrm{pH}$ buffer solution. 

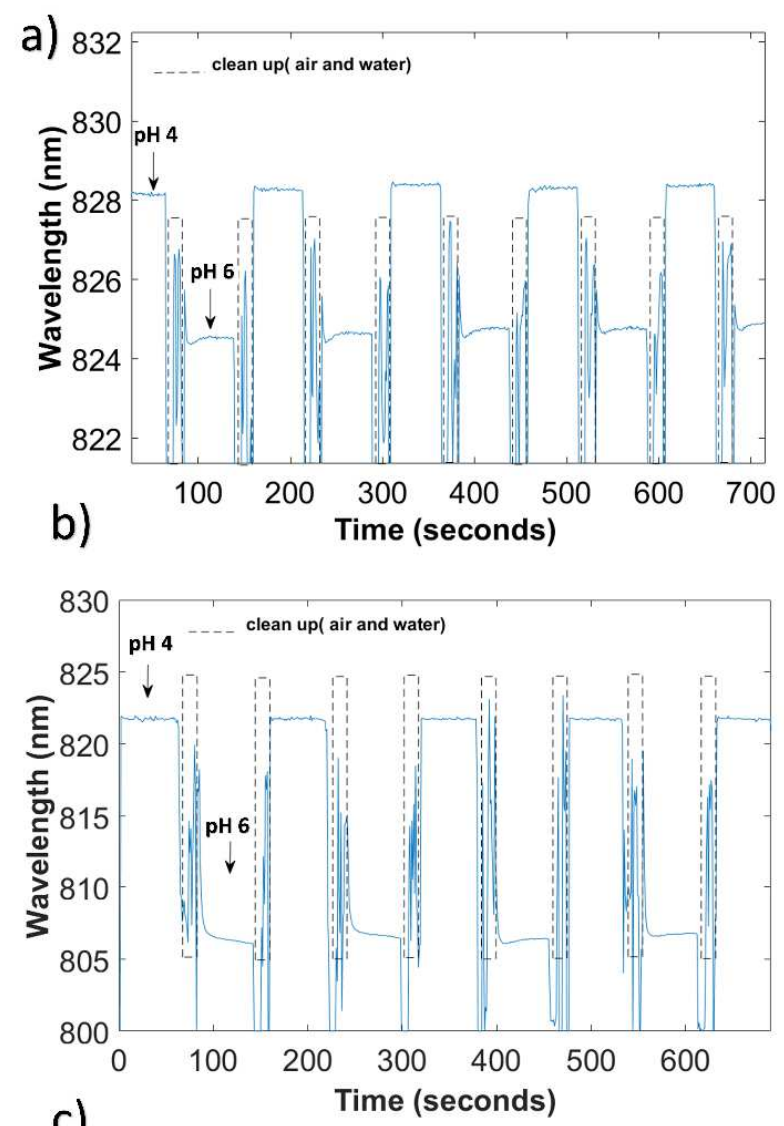

c)

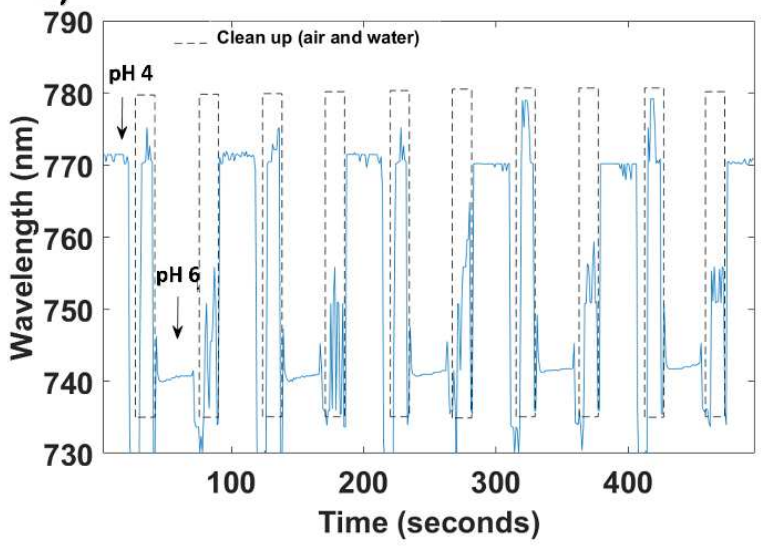

Fig.9 a) E-SMS1 structure sensitivity response. b) E-SMS2 structure sensitivity response. c) E-SMS3 structure sensitivity response.

By comparing the results obtained with the three structures, the E-SMS3 structure improves by a factor of 2 the sensitivity attained with E-SMS2 structure, whereas the E-SMS2 
structure improves by a factor of 3 the sensitivity of E-SMS1 structure. This improvement agrees with previous analysis performed with SMS structures in the telecommunications band $[23,27]$. Moreover, the sensitivity obtained with the E-SMS2 structure equals the best performance of the SMS structure operating in the telecommunications band in [11], whereas the E-SMS3 structure doubles this sensitivity.

The wavelength shift from $\mathrm{pH} 4$ to $\mathrm{pH} 6$ is attributed to the variation of the thickness of the PAH/PAA polymeric matrix, a phenomenon that has been confirmed in other works $[29,30]$. In fact, this phenomenon explains why a hysteresis is observed in the results of Fig. 9, due to the swelling and deswelling of the polymers.

In order to support the idea that the polymer thickness is modified due to the effect of $\mathrm{pH}$, the transmission spectrum for a coating of thickness 40 and $100 \mathrm{~nm}$ has been obtained numerically with FIMMWAVE ${ }^{\circledR}$. The wavelength shift of the transmission band closer to 800 $\mathrm{nm}$ has been analyzed for different diameters of the SMS structure ranging from 20 to 70 $\mu \mathrm{m}$. The selection of the coating thickness values (40 and $100 \mathrm{~nm}$ ), has been based on the estimated thickness of 16-17.5 nm per bilayer obtained in other works [30,31], which should lead to approximately $70 \mathrm{~nm}$ for the 4 bilayers deposited in this work. Considering that the coating swells and deswells as a function of $\mathrm{pH}$, a variation from 40 to $100 \mathrm{~nm}$ has been considered a good option for the analysis. In addition, the refractive index model calculated in [31] has been used here for the PAH/PAA coating.

The model indicates that the sensitivity is inversely proportional to the diameter. The experimental results do not follow exactly the same trend. The sensitivity increase is more abrupt. However, it must be considered that the surface where the PAH/PAA polymeric matrix is deposited presents a lower surface area when the diameter is reduced, which leads to a faster drying of the surface, and it is well know that the bilayer thickness in LBL process is sensitive to the drying step [32-35]. This could be the main reason for the discrepancy between the theoretical and the experimental model. 


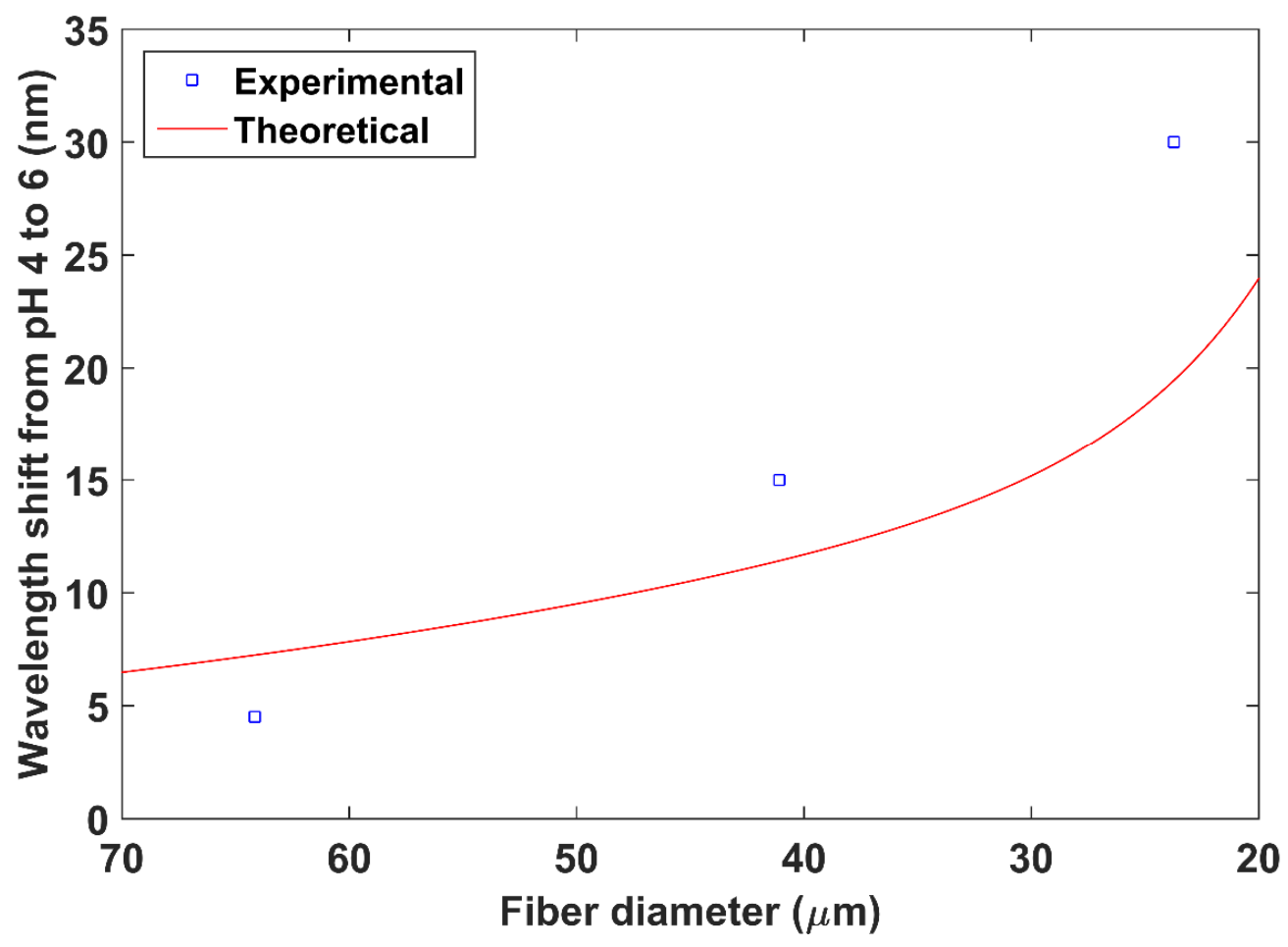

Figure 10: Wavelength shift from $\mathrm{pH} 4$ to $\mathrm{pH} 6$ for different diameters of the SMS structure. The SMS structure was coated with 4 bilayers of PAH/PAA. The theoretical model represents the wavelength shift induced by reducing the nanocoating from $100 \mathrm{~nm}$ to $40 \mathrm{~nm}$.

\section{CONCLUSION}

In this work, it has been demonstrated that the sensitivity of low cutoff SMS etched structures can be improved with an etching process, which can be used for applications operating in the visible and near infrared region, where the cost of optical sources and detectors is reduced compared to the equipment that operates at longer wavelengths.

A pH sensing application has been explored. Initially, the effect of diameter reduction in terms of sensitivity to refractive index was explored. PAH/PAA based sensors can be used for $\mathrm{pH}$ values ranging from 3 to $7[11,28]$. However, in view that the aim in this work is to see the sensitivity increase as a function of the fiber diameter, only $\mathrm{pH}$ values 4 and 6 have been explored. 
On this basis three different etched multimode core diameters were explored for the $\mathrm{pH}$ sensor: $64.15 \mu \mathrm{m}, 41.07 \mu \mathrm{m}, 23.73 \mu \mathrm{m}$. A nanocoating of 4 bilayers, which allowed increasing the sensitivity without compromising the visibility of the transmission bands, was deposited. After this, a maximum wavelength shift of $30 \mathrm{~nm}$ in the $\mathrm{pH}$ range 4-6 was attained with the SMS structure diameter whose diameter was reduced by a factor of 5 compared to the original diameter $(125 \mu \mathrm{m})$.

These results improve the sensitivity attained with unetched SMS structures in the telecommunications band, which opens the door to the development of other applications where the sensitivity is more critical towards the ability of the device to detect a specific parameter, such as biosensors or chemical sensors.

\section{ACKNOWLEDGMENTS}

This work was supported by the Spanish Agencia Estatal de Investigación (AEI) and Fondo Europeo de Desarrollo Regional (FEDER) (TEC2016-79367-C2-2-R, TEC2016-78047-R), Government of Navarra PI044 NANOSEN, Government of Navarra PC023-024 BIPOTSENS research grants and the Direction of Internationalization and Academic Collaboration of the Autonomous University of Tamaulipas.

\section{REFERENCES}

1. E. Budd, W. B. Spillman Jr., Fiber Optic Sensors: An Introduction for Engineers and Scientists, second ed., Wiley, US, 2011.

2. Matias, S. Ikezawa, J. Corres, Fiber Optic Sensors: Status and Future Possibilities, first ed., Springer, Switzerland, 2017.

3. H. Fukano, Y. Kushida, S. Yaue, Sensitivity improvement of optical-fiber temperature sensor with solid cladding material based on multimode interference, Japanese Journal of Applied Pysics, vol. 54(3) (2015) 032502.

4. J. M. López-Higuera, L. Rodríguez Cobo, A. Quintela Incera, A. Cobo, Fiber Optic Sensors in Structural Health Monitoring, Journal of Lightwave Technology, vol. 29 (2015), 587-608. 
5. S. Tripathi, A. Kumar, R. K. Varshney, Y. B. P. Kumar, E. Marin, and J.-P. Meunier, Strain and temperature sensing characteristics of single-mode-multimode-singlemode structures, Journal Lightwave Technology, 27 (2009), 2348-2355.

6. A. Mehta, W. Mohammed, and E. G. Johnson, Multimode interference-based fiberoptic displacement sensor, IEEE Photonics Technology Letters., vol. 15 (8) (2003) , $1129-1131$.

7. Antonio-Lopez, J. E., LiKamWa, P., Sanchez-Mondragon, J. J., and May-Arrioja, D. A., All-fiber multimode interference micro-displacement sensor, Measurement Science and Technology, 24 (2013) 1-7.

8. H. Fukano, D. Watanabe, S. Taue, Sensitivity Characteristics of MultimodeInterference Optical-Fiber Temperature-Sensor with Solid Cladding Material, IEEE Sensors Journal, vol.16 (2016) 8921-8927.

9. A. Rodríguez-Rodríguez, R. Domínguez-Cruz, D. May-Arrioja, I. Matías-Maestro, C. Ruiz-Zamarreño, F. Arregui, MMI fibre optic refractometer with universal $\mathrm{pH}$ indicator coating, IEEE: 4th International Conference on Photonics, Optics and Laser Technology, Photoptics, (2016).

10. F. Chiavaioli, F. Baldini, S. Tombelli, C. Trono and A. Giannetti, Biosensing with optical fiber gratings, Nanophotonics, vol. 6 (2017), 663-679

11. A. B. Socorro, I. Del Villar, J. M. Corres, F. J. Arregui, I. R. Matias, Sensitivity enhancement in a multimode interference-based SMS fibre structure coated with a thin-film: Theoretical and experimental study, Sensors and Actuators B: Chemical, vol. 190 (2014) 363-369.

12. B. Gu, M. Yin, A. P. Zhang, J. Qian, S. He, Biocompatible Fiber-Optic pH Sensor Based on Optical Fiber Modal Interferometer Self-Assembled with Sodium Alginate/Polyethylenimine Coating, IEEE Sensors Journal, vol. 12 (5) (2012), 14771482.

13. S. K. Mishra, B. Zou, K. S. Chiang, Wide-Range pH Sensor Based on a Smart- HydrogelCoated Long-Period Fiber Grating, IEEE Journal of Selected Topics In Quantum Electronics, vol. 23 (2) (2017). 
14. R. Kant, R. Tabassum, B. D. Gupta, Fiber Optic SPR-Based Uric Acid Biosensor Using Uricase Entrapped Polyacrylamide Gel, IEEE Photonics Technology Letters, vol. 28 (19) (2016), 2050-2053.

15. Md. R. Rahaman Khan, A. V. Watekar, and S. Kang, Fiber-Optic Biosensor to detect pH and Glucose, IEEE Sensors Journal, vol. PP (99) (2017).

16. T. H. Nguyen, T. Venugopala, S. Chen, T. Sun, K. T.V. Grattan, S. Taylor, P.A. M. Basheer, A. E. Long, Fluorescence based fibre optic $\mathrm{pH}$ sensor for the $\mathrm{pH} 10-13$ range suitable for corrosion monitoring in concrete structures, Elsevier Sensors and Actuators B: Chemical, vol. 191 (2014), 498-507.

17. A. J. Rodríguez, O. Baldovino-Pantaleón, R.F. Dominguez, C. R. Zamarreño, I. R. Matías, D. A. May-Arrioja, Gasohol Quality Control for Real Time Applications by Means of a Multimode Interference Fiber Sensor, Sensors MDPI, vol. 14 (9) 2014, 17817-17828.

18. A. J. Rodríguez- Rodríguez, R. F. Domínguez-Cruz, D. A. May-Arrioja, Liquid Fuel Identification Using a Fiber Optic Sensor Based in Multimode Interference Effects (MMI), Research in Computing Science, vol. 13, 2017.

19. L. R. Villarreal-Jiménez, A. J. Rodríguez-Rodríguez, S. U. Enríquez-Sías, C. E. González, H. G. Barrón-González, M.J. Erro-Betrán, W.E. Rodríguez-Rodríguez, R.F. DomínguezCruz, An Efficient Optoelectronic System for Remote Salinity Water Sensing, Applied Mechanics and Materials, vol. 876 (2018), 152-160.

20. S. Silva, E. G. P. Pachon, M. A. R. Franco, J. G. Hayashi, F. X. Malcata, O. Frazão, P. Jorge, C. M. B. Cordeiro, "Ultrahigh-sensitivity temperature fiber sensor based on multimode interference", App. Opt., vol. 51, pp. 3236-3242, 2012.

21. A. Rodríguez-Rodríguez, R. Domínguez-Cruz. D. A. May-Arrioja, I. Matías-Maestro, F. Arregui, C. Ruiz-Zamarreño, Fiber Optic Refractometer based in Multimode Interference effects (MMI) Using Indium Tin Oxide (ITO) Coating, Proc.- IEEE Sensors, (2015), 10.1109/ICSENS.2015.7370600. 
22. L. B. Soldano, E. C. M. Pennings, Optical multi-mode interference devices based on self-imaging: principles and applications, Journal of Lightwave Technology, vol. 13 (1995) $615-627$.

23. Y. Cardona-Maya, I. Del Villar, A. B. Socorro, J. M. Corres, I.R. Matias, J. F. BoteroCadavid, Wavelength and phase detection based fibre sensors optimized with etching and nanodeposition, Journal of Lightwave Technology IEEE, (2017), 3743 3749.

24. A. B. Socorro, I. Del Villar, J. M. Corres, F. J. Arregui, I. R. Matias, Mode transition in complex refractive index coated single-mode-multimode-single-mode structure, Opt. Express, 21(19) (2013) 12668-12682.

25. I. Del Villar, J. L. Cruz, A.B. Socorro, J. M. Corres, And I.R. Matias, Sensitivity optimization with cladding-etched long period fiber gratings at the dispersion turning point, Optics Express, vol. 24 (16) (2016), 17680-17685.

26. F. Chiavaioli, C. A. J. Gouveia, P. A. S. Jorge, F. Baldini, Towards a Uniform Metrological Assessment of Grating-Based Optical Fiber Sensors: From Refractometers to Biosensors, MDPI Biosensors, vol. 7(2) (2017).

27. I. Del Villar, A. B. Socorro, J. M. Corres, F. J. Arregui, I. R. Matias, Refractometric sensors based on multimode interference in a thin-film coated single-modemultimode-single-mode structure with reflection configuration, Appl. Opt., 53(18) (2014) 3913-3919.

28. C. R. Zamarreño, M. Hernáez, I. Del Villar, I. R. Matías, F. J. Arregui, Optical fiber pH sensor based on lossy-mode resonances by means of thin polymeric coatings, Sensors and Actuators B: Chemical, vol. 155 (1) (2011) 290-297.

29. J, Choi, M. F. Rubner, Influence of the Degree of Ionization on Weak Polyelectrolyte Multilayer Assembly, ACS Publications: Macromolecules, vol. 38(1) (2005) 116-124.

30. J, Goicoechea, C.R.Zamarreño, I.R.Matias, F.J. Arregui, Utilization of white light interferometry in $\mathrm{pH}$ sensing applications by mean of the fabrication of nanostructured cavities, Elsevier: Sensors and Actuators B, Vol. 138 (2009) 613-618. 
31. I. Del Villar, M. Hernaez, C. R. Zamarreño, P. Sánchez, C. Fernández-Valdivielso, F. J. Arregui, and I. R. Matias, Design rules for lossy mode resonance based sensors, OSA Publishing: Applied Optics, vol. 51 (19) (2012) 4298-4307.

32. J. Chen, G. Luo, W. Cao, "The Study of Layer-by-Layer Ultrathin Films by the Dynamic Contact Angle Method," J. Colloid Interface Sci., vol. 238 (2001) 62-69.

33. Y. Lvov, K. Ariga, M. Onda, I. Ichinose, T. Kunitake, "A careful examination of the adsorption step in the alternate layer-by-layer assembly of linear polyanion and polycation," Colloids Surf. A, vol. 146 (1999), 337-346.

34. D. S. Patel, R. K. Aithal, G. Krishna, Y. M. Lvov, M. Tien, D. Knila, "Nano-assembly of manganese peroxidase and lignin peroxidase from P. chrysosporium for biocatalysis in aqueous and non-aqueous media," Colloids Surf. B Biointerfaces, vol. 43 (2005), 13-19.

35. L. Zhang, H. Liu, E. Zhao, L. Qiu, J. Sun, J. Shen, Drying and Nondrying "Layer-by-Layer Assembly for the Fabrication of Sodium Silicate/TiO2 Nanoparticle Composite Films," Langmuir, vol. 28 (2012), 1816-1823. 